\title{
Enhancing IEEE 802.11a/n with Dynamic Single-User OFDM Adaptation
}

\author{
James Gross ${ }^{\mathrm{a}, *}$, Marc Emmelmann ${ }^{\mathrm{b}, * *}$, Oscar Puñal ${ }^{\mathrm{a}, *}$, \\ Adam Wolisz ${ }^{\mathrm{b}, * *}$ \\ ${ }^{a}$ Mobile Network Performance Group, UMIC Research Centre, RWTH Aachen University, \\ Germany \\ ${ }^{\mathrm{b}}$ Telecommunication Network Group, Technical University Berlin, Germany
}

\begin{abstract}
Earlier paper have demonstrated that the achievable throughput of OFDM systems can benefit significantly from individual modulation/transmit power selection on a per sub-carrier basis according to the actual gain of individual sub-carriers (so called dynamic OFDM scheme). Usage of such approach requires, however, providing support for additional functionalities like: acquisition of the sub-carrier gains, signaling of the used modulation types between sender and receiver, etc. Therefore dynamic OFDM is actively pursued for future radio interfaces, rather than considered as extension of existing OFDM based standards. In this paper we introduce a proposal how the widely accepted IEEE $802.11 \mathrm{a} / \mathrm{g}$ systems as well as the emerging IEEE $802.11 \mathrm{n}$ system might be extended to support the dynamic OFDM in a single-user (point-to-point) setting. The presented approach guarantees backward compatibility to legacy devices. We address these issues by presenting a) a set of protocol modifications required to incorporate dynamic OFDM in $802.11 \mathrm{a} / \mathrm{g} / \mathrm{n}$; and b) a performance evaluation of the suggested extension (referred further on to as single-user 802.11 DYN mode). Although 802.11n already includes advanced MAC and PHY features, i.e., frame aggregation and MIMO transmissions, our performance evaluation demonstrates that a further improvement is achievable by incorporating dynamic OFDM.
\end{abstract}

Key words: WLAN, IEEE 802.11, dynamic OFDM, adaptive modulation

\footnotetext{
* UMIC Research Centre, RWTH Aachen University, Templergraben 55, 52065 Aachen, Germany

** Technical University Berlin, Sekr. FT-5, Einsteinufer 25, 10587 Berlin, Germany

Email addresses: gross@umic.rwth-aachen. de (James Gross), emmelmann@ieee.org (Marc Emmelmann), punal@umic.rwth-aachen. de (Oscar Puñal), wolisz@tkn.tu-berlin. de (Adam Wolisz).
} 


\section{Introduction}

IEEE 802.11 wireless local area networks are almost omnipresent today and are expected to proliferate further in the future. Hence, the research and standardization activity in this field has become quite intense, addressing a wide range of issues like security (IEEE 802.11i [6]), quality of service (IEEE 802.11e [7]), and inter-access point coordination (IEEE 802.11F [4]).

Among these, increasing the throughput of the available channel is one major issue and research has been mainly focused on improving the modulation and coding within the Physical Layer. From the initial DSSS with up to $2 \mathrm{Mbit} / \mathrm{s}$ in the 1999 version of the IEEE 802.11 standard [1], IEEE 802.11b provided up to $11 \mathrm{Mbit} / \mathrm{s}$ via complementary code keying (CCK) modulation and DSSS packet binary convolutional coding (PBCC) [2]. Finally, IEEE 802.11a/g achieved up to $54 \mathrm{Mbit} / \mathrm{s}$ by employing orthogonal frequency division multiplexing (OFDM) in combination with high-rate signal constellations [3,5]. This huge performance jump-even if achieved only for very limited distances-is due to the inherent features of OFDM. While the scheme itself is known for over thirty years [19], its features have become especially attractive for high rate, broadband systems. In OFDM, the system bandwidth given is split into many sub-channels, also referred to as sub-carriers. Instead of transmitting symbols sequentially through one channel, multiple symbols are transmitted in parallel. This leads to much longer symbol durations, such that the impact of inter-symbol interference decreases significantly. Therefore, no additional measures like a costly equalization are necessary [29]. Today, OFDM is used as foundation of most high speed standards, e.g., digital audio and video broadcasting [12] and the most recent amendment of IEEE 802.11 that will provide "high throughput" of up to $600 \mathrm{Mbit} / \mathrm{s}$ [10], while it is a strong candidate for several upcoming standards (3rd generation broadband evolution, for example). With regard to WLANs, however, the potential of further bit-rate increase is usually not seen in improving the way in which OFDM is used in IEEE $802.11 \mathrm{a} / \mathrm{b} / \mathrm{g}$, but rather in the introduction of channel bonding, using multiple-input multipleoutput (MIMO) antenna systems, or improving the efficiency of the MAC itself as followed in 802.11n [8,31,10].

In this paper, we suggest in addition to these measures a possibility of increasing the bit-rate achievable from any given channelization by using the concept of the so called dynamic OFDM introduced in [14] around 1990. Dynamic OFDM is based on the observation that the gain of individual sub-carriers of an OFDM channel is variable in time and frequency-i.e., in any given time epoch the individual subcarriers do not have an identical gain. Thus, it has been clearly demonstrated that the performance in terms of throughput, power consumption, error behavior, etc. of an OFDM link (i.e., a single-user, point-to-point connection) can be improved by adapting the transmit power and/or the modulation type to the current gain of each sub-carrier. Such schemes are often referred to as loading algorithms [17,32]. One 
particular simple but still very efficient dynamic scheme is adaptive modulation, where the transmit power per sub-carrier is fixed and only the modulation type per sub-carrier is varied according to the SNR. In fact, in [21] it has been shown that adapting the modulation while keeping the transmit power fixed provides a large performance boost which is only marginally improved by also adapting the transmit power.

The performance gain from loading algorithms comes at some cost system wise. Obviously, without an accurate estimate of the sub-carrier gains these dynamic schemes cannot be applied by a transmitter. Acquiring the sub-carrier states consumes system resources, i.e., time, power, and bandwidth. Second, computational resources are required at the transmitter to generate the dynamic adaptation. A lot of research within the OFDM community has focused on this issue. Third, the receiver has to be informed of the current "assignments" per sub-carrier (i.e., in case of the adaptive modulation the modulation type used per sub-carrier); otherwise it cannot decode the data correctly. The need to support all the above mentioned features resulted in dynamic OFDM being intensively considered for future standards, but not being taken into consideration as possible enhancement of already deployed systems. In fact, todays OFDM-based IEEE 802.11 applies some simple (manufacturer proprietary) mechanisms for rate adaptation to variable channel conditions (referred to as link adaptation). However, these schemes adapt the modulation over the whole set of sub-carriers equally and do not take the individual sub-carrier gains into account.

This paper is an extended version of [26] proposing a complete concept for introducing the dynamic, per sub-carrier adaptation for the IEEE $802.11 \mathrm{a} / \mathrm{g}$ systems, which we denote in the following as (single-user mode of) 802.11 DYN. Our major contribution consists of: (a) demonstrating that a proper support for dynamic OFDM can be built into the actual IEEE $802.11 \mathrm{a} / \mathrm{g}$ standard, while supporting full backward compatibility; and (b) providing simulative performance evaluation of the proposed dynamic OFDM with per-sub-carrier modulation adaptability, taking into consideration all the necessary protocol overhead. In addition, we describe how to (c) incorporate dynamic OFDM into the "high throughput" amendment IEEE 802.11n and evaluate its performance for two novel features incorporated in $11 n$, i.e., frame aggregation and advanced MIMO systems enabling multiple spatial streams for data transmission. Hence, we herein provide for the first time a comparative investigation of our novel dynamic scheme with the next generation, emerging WLAN technology.

The remaining paper is organized as follows. In Section 2 we provide an (highlevel) overview of the existing IEEE $802.11 \mathrm{a} / \mathrm{g}$ standard and the enhancements added by the high throughput amendment 802.11 n. Furthermore, we discuss some related work on adaptation to varying channel conditions in the context of WLAN. Next, in Section 3, we define the new transmission scheme for dynamic OFDM and present the concepts featuring its support. Then, in Section 4, we evaluate the 
performance of this new scheme (in combination with the suggested protocol extensions) and compare it to legacy IEEE 802.11a and 802.11n with and without usage of RTS/CTS. Finally, in Section 5, we comment on conclusions and future work.

\section{Overview of IEEE 802.11 WLAN}

This section summarizes those MAC and PHY layer aspects of the OFDM-based IEEE 802.11 standard which either have to be amended to incorporate dynamic OFDM or are used to enable downward compatibility of the enhancements with existing legacy IEEE 802.11 devices. For a detailed discussion the reader might refer to $[24]$ or to the standard itself $[9,10]$.

\subsection{IEEE 802.11 Architecture and Medium Access Scheme}

IEEE 802.11 stations (STA) may either communicate directly with each other in an "ad-hoc" mode forming an independent basic service set (iBSS) or via an access point (AP) forming an infrastructure basic service set (BSS). APs announce the existence of a BSS by regularly transmitting beacons including a capability information field which contains, e.g., information regarding all supported PHY rates/modulation types [9].

The mandatory medium access schema for IEEE 802.11 is the distributed coordination function (DCF) which employs carrier sense multiple access with collision avoidance and binary exponential back-off (CSMA/CA). STAs refrain from transmitting if either physical or virtual carrier sensing indicate the wireless media (WM) occupied. The latter is realized using the network allocation vector (NAV). The NAV is set according to the duration field found in the MAC header of every packet. In particular, the RTS/CTS handshake preceding the transmission of the data packet employs this mechanism to exclusively reserve the medium by usually indicating the remaining time until the ongoing transmission (sequence) is finished [9].

\subsection{IEEE $802.11 \mathrm{a} / \mathrm{g}$}

Amendment IEEE 802.11a [3,9] as well as the extended rate PHY (ERP) of IEEE 802.11g [5,9] employ OFDM physical layers to provide data rates up to $54 \mathrm{Mbit} / \mathrm{s}$ in the $5 \mathrm{GHz}$ and $2.4 \mathrm{GHz}$ band correspondingly. The available bandwidth is divided into 52 sub-carriers from which four are exclusively used as pilots. Both 
OFDM-based amendments utilize link adaptation. For a payload data transmission the data is first convolutional encoded. The resulting data block is transmitted via all 48 sub-carriers employing the same modulation type on each sub-carrier. Eight different modulation/coding modes are available, i.e.: combining BPSK, QPSK, 16-QAM and 64-QAM with either rate $1 / 2,3 / 4$, or $3 / 4$ coding. The choice of the employed "mode" is crucial for the performance but not standardized. Instead, the MAC may make usage of, e.g., the radio signal strength indicator (RSSI) level gained during reception of previous packets or adapt the rate depending on the success of a block transmission.

The modulation scheme (mode) employed for the PHY service data unit (SDU) of a particular transmission is signaled to the receiver via the PLCP header's rate field which is always transmitted using mode 1 (BPSK with rate $1 / 2$ ). STAs not supporting the indicated rate may hence discard the remainder of the received frame.

\subsection{IEEE 802.11n}

Recent standardization activities within the IEEE (under the task group label 802.11n) concentrate on an even faster WLAN version to achieve transmission rates up to 600 $\mathrm{Mbit} / \mathrm{s}$. This is accomplished by a variety of different improvements on the physical layer as well as on the medium access layer [10]. In the following we give a brief overview of some of these new features mentioned later on in this paper.

The major improvement on the medium access layer is frame aggregation. Applying this technique, several payload packets are transmitted during one MAC frame time, i.e. within one channel access in 802.11. Obviously, this improves the efficiency as the overhead for framing and channel access is only spent once. On the other hand frame aggregation is more sensitive to interference as the medium is blocked for a longer time by a single (aggregate) data transmission. The IEEE 802.11n draft suggests two different ways of performing frame aggregation: aggregated MSDU (A-MSDU) and aggregated MPDU (A-MPDU). The first performs the aggregation at the "top of the MAC" (thus aggregating MAC SDUs without 802.11 specific framing) while the second one performs aggregation "below the MAC" (thus aggregating several payload packets each with a separate MAC header). Note that even in the case of A-MPDU only packets with an identical destination address can be aggregated. Clearly, A-MSDU reduces the overhead to a minimum at the cost of an increased packet error probability. In contrast, A-MPDU enables to check each single packet for an error (by the CRC) while featuring a higher overhead. In addition, A-MPDU enables the usage of block acknowledgments, requesting the retransmission of only a few (or even only one MPDU) out of the set of all transmitted MPDUs. Both frame aggregation types have a maximal data aggregation size: $8 \mathrm{kByte}$ in case of the A-MSDU and $64 \mathrm{kByte}$ in case of the A-MPDU type. 


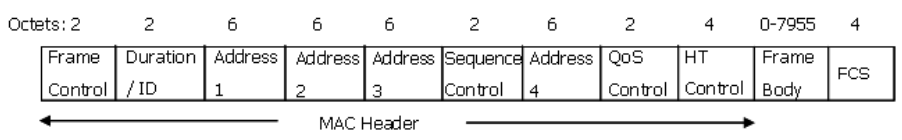

Fig. 1. MAC frame format of 802.11n, [10, Cls. 7.1.2].

In the physical layer, several improvements are suggested. The most significant one is the introduction of multiple-antenna capabilities at the transmitter and receiver side. Specifically, these can be distinguished into transmit beamforming, spatial multiplexing and space-time-coding. Transmit beamforming enables a directional shaping of the transmit signal and is mainly used to reduce the impact due to fading at the receiver antenna (while also reducing the interference impact to other cells outside the direction of the current beam). Spatial multiplexing enables the transmission of several different data flows over each of the antennas (without requiring more radio spectrum). Finally, space-time coding uses the additional degree of freedom (provided by the antennas) for improving primarily the error probability (thus, not doubling or tripling the raw data rate as such). Note that in the case of transmit beamforming, the transmitter has to acquire the channel knowledge while in case of spatial multiplexing at least the receiver has to do so (however, if the transmitter also acquires the channel state performance can be even more boosted). In addition to these MIMO capabilities, IEEE 802.11n also specifies a slightly higher number of sub-carriers to be used (56 instead of 52), an optional shorter guard period (set to $0.4 \mu \mathrm{s}$ ), state-of-the-art error correction coding (as well as featuring convolutional coding of rate $5 / 6$ ), and channel bonding (an optional technique to increase the used bandwidth by a factor of two). The general MAC frame format in IEEE $802.11 \mathrm{n}$ is shown in Figure 1. There are two basic changes compared to legacy 802.11, namely the QoS Control field ( 2 bytes) and the High Throughput Control (HT Control) field (4 bytes), increasing the overall MAC header by 6 bytes. The HT Control field is responsible for carrying important $\mathrm{PHY}$ and MAC information regarding link adaptation, antenna selection and calibration among other information. Moreover, IEEE $802.11 \mathrm{n}$ adds also some changes to the PLCP preamble. The format of the preamble depends on whether there are only $802.11 \mathrm{n}$ stations involved in the communication (green field mode), only legacy 802.11 stations, or both of them (mixed mode). The green field mode is optional, while the other two are mandatory. In the mixed and the greenfield mode, the High Throughput Long Training field (HT-LTF) is used to train the MIMO structures of the receiver, hence providing it with information to estimate the channel. The number of training symbols must be equal or greater than the number of space-time streams.

\section{Dynamic OFDM for IEEE 802.11}

In this section we first review the concept of dynamic OFDM for point-to-point, i.e. single-user, connections and discuss the system requirements related to it in 
general. Then we present our proposal how to modify the existing IEEE 802.11a/g standard such that it can benefit from a dynamic single-user OFDM mode. A more detailed presentation of this work can be found in [24], a short presentation of this work has also been given to the IEEE 802.11 standardization committee [25]. Finally, we extend our proposal for IEEE 802.11n networks, especially considering the case of spatial multiplexing in the physical layer.

\subsection{Dynamic Single-User OFDM}

Consider the following situation: A packet of length $\varsigma$ bits is to be transmitted via an OFDM link with $N$ sub-carriers. For the transmission a maximum power of $P_{\max }$ is available. Each sub-carrier $n$ has a certain channel gain $h_{n}^{2}$ during the transmission. The channel gain varies due to several effects, most importantly it varies in time as well as in frequency due to fading. The bandwidth of the OFDM system is large, hence, over the set of the $N$ sub-carriers the channel gains vary strongly. Compared to the average channel gain of the link, i.e. $\overline{h^{2}}=1 / N \sum_{\forall n} h_{n}^{2}$, there are always several sub-carriers which are in a bad fading state. We will further assume that at the beginning of each packet transmission, the precise gain for each sub-carrier is known, and will remain constant over the time needed for the transmission of the entire packet.

Dynamic OFDM is defined as a family of approaches in which the transmitter adaptively controls the modulation type, the transmit power and the coding scheme applied on a per packet and per sub-carrier basis, in order to adjust itself in the best possible way to the actual sub-carrier gains. Several different strategies can be applied. Bit loading $[28,32,15]$ refers to the case where the transmitter maximizes the sum data rate over all sub-carriers by varying the transmit power $p_{n}$ and modulation assignment $r_{n}$ per sub-carrier. It requires (as input) a maximum transmit power budget $P_{\max }$ as well as a target bit error rate (BER) $p_{\max }$. Given a certain target bit error rate, each modulation type $m$ (out of the set of $M$ overall available types) of the transmission system can only be used from a certain signal-to-noise ratio (SNR) switching point $\Gamma_{m}$ on. If the SNR is below that switching point, modulation type $m$ produces too many errors.

A somewhat simpler scheme to apply is adaptive modulation. In adaptive modulation the transmitter assigns each sub-carrier the same transmit power $p_{n}=P_{\max } / N$. Together with the channel gain $h_{n}^{2}$, this results in a specific SNR value $\gamma_{n}$ per subcarrier. Given this SNR value per sub-carrier and the target BER, the transmitter applies the best modulation type to each sub-carrier with respect to the target BER. As the SNR per sub-carrier varies (from packet to packet), the applied modulation type per sub-carrier varies too. The choice of the target BER has obviously quite an impact, as a lower target BER leads to higher SNR switching points per modulation type (and therefore to a lower physical layer throughput). Refer to [21] for 


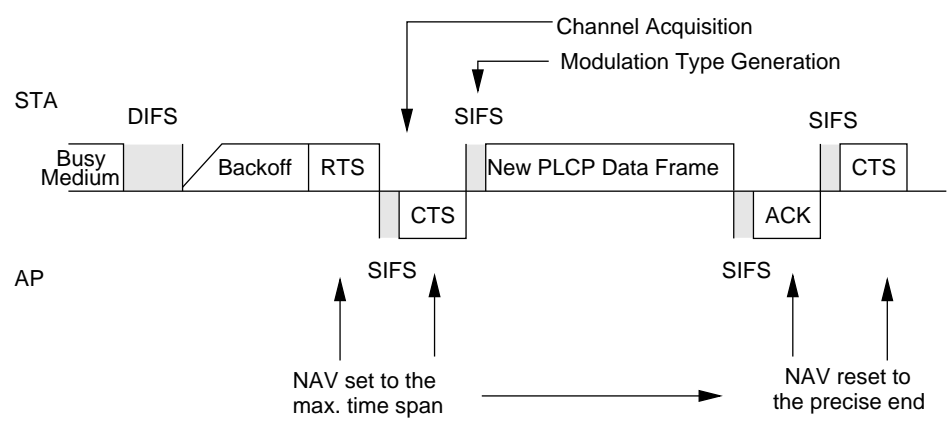

Fig. 2. Transmission sequence of 802.11 DYN.

an detailed discussion of the performance difference between adaptive modulation and bit loading.

We suggest to apply dynamic OFDM to the payload part of packet transmission in IEEE 802.11a/g WLANs (for the infrastructure as well as for the ad-hoc mode). Both the above discussed schemes for dynamic OFDM are feasible only if three specific requirements are fulfilled: First of all, the transmitter has to acquire information about the current sub-carrier gains. Second, the transmitter has to perform some computation of the sub-carrier adaptations depending on the channel information. Third, the receiver has to be informed of the used modulation type per subcarrier in order to decode the information correctly. As IEEE 802.11a/g does not support any of the above formulated requirements, the standard has to be modified to assure such support. The suggested modifications should be as simple as possible, and the backward compatibility with existing equipment should be assured-so that operating a mixture of dynamic OFDM enhanced stations and "legacy" stations is feasible.

\subsection{Dynamic OFDM on Top of $802.11 \mathrm{a} / \mathrm{g}$}

In the following we present our concept for 802.11 DYN - a modification of the IEEE $802.11 \mathrm{a} / \mathrm{g}$ standard supporting dynamic OFDM. While this is one possible way how this goal can be achieved, we believe that our proposal offers the desired support in a consistent and rather easy-to-implement way.

The first issue to be addressed is how the transmitter can obtain the channel knowledge, i.e., the current gain per sub-carrier. As solution we suggest for 802.11 DYN a mandatory usage of the RTS/CTS handshake prior to a transmission in the dynamic OFDM modus. According to the IEEE 802.11 standard this is not mandatory. However, by receiving a CTS the transmitter can estimate the channel based on the PLCP preamble. This is possible as the wireless channel has been shown to be reciprocal, i.e., the channel gain from the transmitter to the receiver is equivalent to the one from the receiver to the transmitter [13]. So in 802.11 DYN the transmitter has to decide about usage/non-usage of the dynamic modus on a per packet 


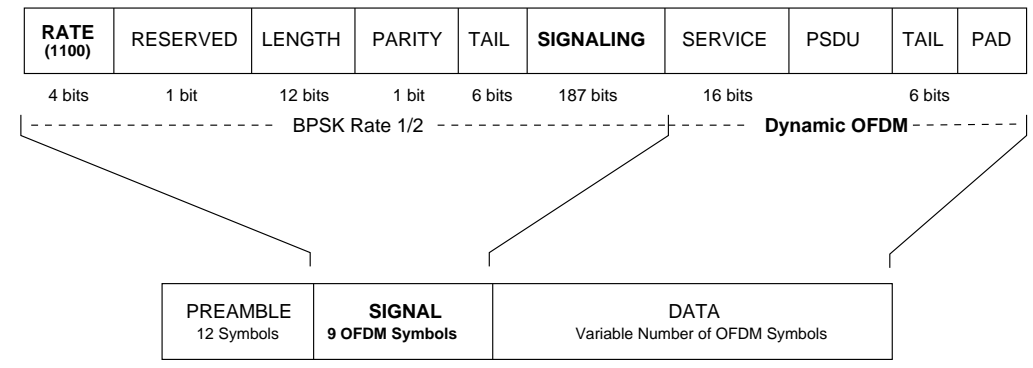

Fig. 3. Structure of the 802.11 DYN PLCP frame (based on the PHY of 802.11a).

basis. In detail, the transmitter starts a dynamic single-user OFDM packet transmission by conveying a normal RTS packet, using exactly the same framing as in IEEE $802.11 \mathrm{a} / \mathrm{g}$ (see Figure 2). After the duration of a SIFS, i.e. $16 \mu \mathrm{s}$, the receiver replies with a CTS frame, also transmitted in accordance to IEEE $802.11 \mathrm{a} / \mathrm{g}$. Based on the channel state information obtained from this CTS frame (specifically from the preamble of the CTS frame), the transmitter generates the appropriate modulation assignments per sub-carrier (either by applying adaptive modulation or by applying bit loading).

Next comes the modified payload transmission. Any 802.11 DYN payload frame uses a modified header of the physical layer convergence protocol (PLCP) frame such that the receiving station can distinguish between legacy IEEE 802.11a/g transmissions and 802.11 DYN transmissions (c.f. Fig. 3). This modified PLCP header starts with a usual PLCP preamble. Next, the new PLCP header is transmitted. The first 24 bits of this header are in total compliance to legacy IEEE $802.11 \mathrm{a} / \mathrm{g}$, with the exception that in the Rate field a different bit sequence is inserted, which is not specified in legacy IEEE $802.11 \mathrm{a} / \mathrm{g}$. We propose the bit sequence 1100 as identification that the following data transmission is compliant to $802.11 \mathrm{DYN}$; legacy 802.11 stations simply ignore the remainder of the transmission at this point as an "unsupported rate" is indicated. After the Tail field a new element of the header is transmitted, the Signaling field. This field contains all the information to decode the following payload transmission according to 802.11 DYN. The layout of the signaling field is discussed in detail below. After the Signaling field, the Service field is added (which has the same layout and interpretation as in legacy IEEE 802.11a/g systems), then the protocol service data unit (PSDU) is conveyed containing the IEEE 802.11 MAC packet with the payload. The complete new PLCP header is transmitted applying the BPSK modulation type and the rate $1 / 2$ convolutional coding. Compared to legacy IEEE $802.11 \mathrm{a} / \mathrm{g}$ systems, the header is only longer by the number of octets required for the Signaling field.

A particular problem with 802.11 DYN arises from managing the NAV. In legacy transmissions, the transmitter knows already the duration of the data frame when conveying the RTS frame. However, as dynamic OFDM adapts to the sub-carrier states, which are only known after reception of the CTS, a new approach has to be taken. At the initial RTS frame the NAV is set to the longest possible transmission duration which would be required by worst channel characteristics. Hence, the CTS 


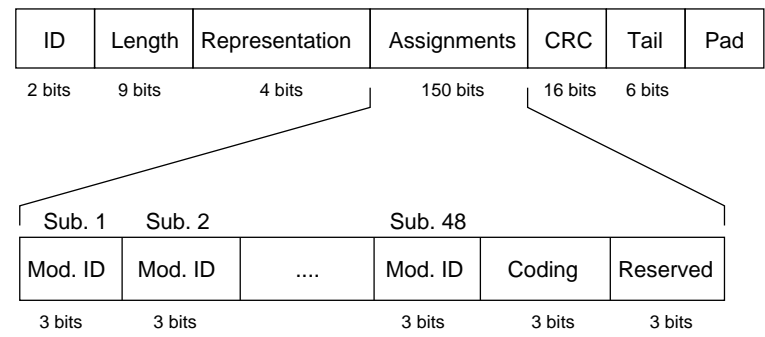

Fig. 4. Structure of the 802.11 DYN Signaling field (based on the PHY of 802.11a).

frame will also announce this duration. After computing the correct length of the data transmission, the transmitter sets the correct value of the NAV. As this correct setting is only part of the MAC packet and the MAC packet is part of the new PLCP packet, legacy stations will not receive the corrected NAV setting (legacy NICs discard the 802.11 DYN PLCP frame after decoding a wrong Rate field). Therefore, the frame exchange after the payload transmission has to be modified such that all stations can finally set the NAV to the correct value. We suggest that after the dynamic OFDM payload transmission, the ACK frame resets the NAV to a value just long enough to cover a new CTS frame addressed to (and transmitted by) the initiator itself. This finally sets the NAV to zero, releasing the WM, and ensures that the NAV is set to the correct value for all listening stations.

Furthermore, let us focus here on two specific issues: The generation of modulation types per sub-carrier and the exact layout of the signaling field. An important issue related to the generation of the modulation types per sub-carrier is the execution time. Note that once the PLCP preamble of the (first) CTS frame is received, the transmitter has to generate the assignments together with the PLCP header within $36 \mu \mathrm{s}$ (the remaining CTS frame requires $20 \mu \mathrm{s}$, then follows a SIFS, which has a duration of $16 \mu \mathrm{s}$ ). If the generation of the sub-carrier assignments requires more than $54 \mu \mathrm{s}$ (which contains the remaining CTS frame plus a DIFS), other stations may start acquiring the channel as they might believe the medium is idle (nothing has been transmitted during a time span of a DIFS from the end of the last CTS frame symbol, assuming these stations have not received the NAV setting previously). If this is the case, a busy tone could prevent this event. However, there is evidence that the modulation types can be generated within the $36 \mu$ s using standard hardware [32]. Certainly, if only adaptive modulation is applied while the transmit power is kept fixed, the modulation types can be determined within the above time span.

We suggest the following formats for the Signaling field. Initially, an ID field is transmitted with 2 bit in length (in case that the specific Rate field bit combination 1100 is used by other extensions to IEEE $802.11 \mathrm{a} / \mathrm{g}$ as well). Next, a Length field of 9 bit is inserted, which indicates the entire size of the Signaling field. The third field is the Representation field. It is 4 bit long and indicates primarily different types of representing the signaling information (for example, compressed signaling information). Then, the information about the modulation type per sub-carrier fol- 
lows in the Assignment field. The modulation types have to be encoded using 3 bits, as it might also happen that a sub-carrier is not utilized at all, i.e. is not allocated any power or modulation type (so there are five modulation types). One example representation of the assignment information is the following. The binary modulation type identifiers are transmitted sequentially without any further delimiter. The position of each identifier in the bit stream corresponds then to the sub-carrier. At the end of the Assignment field 6 more bits are transmitted indicating the used coding scheme as well as 3 bit for a reserved field. Finally, a 16 bit CRC and a 6 bit tail are transmitted at the end of the signaling field. In total, the signaling field is 187 uncoded bits long (which equals 8 OFDM symbols for the transmission of the coded field). As indicated above, the length of this field could be decreased by the usage of compression schemes for the assignment information [30]. In order to indicate this to the receiver, enough combinations are left in the Representation field. In total, the new PLCP header is longer by these 8 OFDM symbols which equals a time span of $32 \mu \mathrm{s}$.

How do stations and APs identify that their communication peer supports 802.11 DYN? For the infrastructure mode, we suggest the following solution. APs announce their support of 802.11 DYN in a special capability field of the Beacon. If a station receives such a Beacon, it will trigger 802.11 DYN the first time it transmits a data frame to the AP. Then the AP is informed of the 802.11 DYN support by the station and stores this information in a list of currently associated stations.

\subsection{Dynamic OFDM on Top of 802.11 n}

In order to add dynamic OFDM to $802.11 \mathrm{n}$, further changes have to be made in comparison to the above mentioned concept for $802.11 \mathrm{a} / \mathrm{g}$. These changes stem primarily from the usage of multiple antennas in case of 802.11n. In the following we focus exclusively on the usage of spatial multiplexing. In this case, several different data streams are transmitted over the different antennas without using channel knowledge at the transmitter for beamforming. By adding dynamic OFDM features, the transmitter adapts the modulation type per sub-carrier and spatial stream. This requires obviously channel knowledge at the transmitter which is acquired by a similar method as presented above using the RTS/CTS handshake. Note that alternatively the transmitter could acquire channel knowledge to apply beamformingwith or without adapting the modulation types per sub-carrier. However, due to space limitations we consider in this paper only spatial multiplexing with dynamic OFDM.

Let us start with the acquisition of the channel knowledge. As mentioned above, the transmitter and receiver perform a mandatory RTS/CTS handshake. In case that several transmit antennas are available at both peers, during the preamble of the RTS and CTS frame each antenna is trained separately by the High-Throughput 
Long Training Field. Once the transmitter has acquired the channel, it adapts the modulation types per sub-carrier and spatial stream. Then, the payload packet is transmitted together with the signaling information. The format of the signaling field remains the same, however, the number of total sub-carriers increases as it is higher anyway in $802.11 \mathrm{n}$ (52 instead of 48 payload sub-carriers) and several spatial streams might be active (multiplying the number of sub-carriers by the number of spatial streams). For example, for a 2 by 2 antenna system, the total length of the signaling information increases now to 358 bits, as the system features 104 subcarriers each requiring 3 bit of signaling information, plus the indication for the coding used per spatial stream (in total 6 bits) plus all the other fields of Figure 4. Next, the payload is transmitted via the two spatial streams. Finally, the channel is cleared by the acknowledgment and the CTS-to-self frame (without any modifications of the 802.11 DYN protocol discussed above). Thus, the main modifications stem from an additional overhead for acquiring the channel knowledge per spatial stream during the RTS/CTS handshake and for signaling the dynamic adaptations per sub-carrier and spatial stream.

\section{Performance Evaluation}

We have evaluated 802.11 DYN in comparison to legacy $802.11 \mathrm{a} / \mathrm{g}$ as well as $802.11 \mathrm{n}$ by means of simulation. When comparing $802.11 \mathrm{DYN}$ with $802.11 \mathrm{a}$, we refer to dynamic OFDM on top of 802.11a, as presented in Section 3.2. When comparing 802.11 DYN to 802.11n, we refer by 802.11 DYN to the concept as discussed in Section 3.3. To make this point clear, in the following we will talk of 802.11 DYN/a and 802.11 DYN/n respectively. In general, we have focused only on the DCF infrastructure mode of IEEE 802.11. Next, we first focus on the investigation regarding 802.11a, afterwards we discuss the combination with $802.11 \mathrm{n}$.

\subsection{Comparison of 802.11 DYN/a and 802.11a}

\subsubsection{Simulation Model and Methodology}

We consider a rather simple set-up, consisting of one access point and one station. The access point is assumed to have always a packet to be transmitted (saturation mode). The packets (which are MAC PDUs, hence, having a MAC header) have a fixed size of $\varsigma$ bits. The maximum transmit power equals $P_{\max }=10 \mathrm{~mW}$. The bandwidth, the number of sub-carriers, the symbol duration and the guard interval are all chosen in accordance to IEEE 802.11a (see Section 2.2).

The sub-carrier gains $h_{n}^{(t)}$ are generated based on path loss and fading. For the path loss, a standard model $h_{\mathrm{pl}}^{2}=K \cdot \frac{1}{d^{\alpha}}$ is assumed [18], parameterized by 
$10 \log K=-46.7 \mathrm{~dB}$ and $\alpha=2.4$ (corresponding to a large office space propagation environment). The fading samples $h_{\text {fad }}^{2}$ correspond to a Rayleigh fading nonline-of-sight scenario with a rms delay spread of $100 \mathrm{~ns}$. A trace of several thousand such samples is generated and for each packet transmission a set of (frequency-) correlated fading coefficients is randomly drawn. Therefore, we do not consider the correlation in time, only the one in frequency. Also, the sub-carrier gains are assumed to be stable during the transmission of a complete PLCP frame - either in legacy mode or in the dynamic OFDM mode [13]. The noise power $\sigma^{2}$ is computed at an average temperature of $20^{\circ} \mathrm{C}$ over the bandwidth of a sub-carrier.

As primary metric we consider the average goodput in bits per second at the link layer. Three different schemes are compared:

(1) Legacy IEEE 802.11a without RTS/CTS handshake.

(2) Legacy IEEE 802.11a with RTS/CTS handshake.

(3) Dynamic OFDM according to 802.11 DYN/a with adaptive modulation and equally distributed transmit power.

We consider for the two legacy schemes the performance of each physical layer mode (the eight different combinations of coding scheme and modulation type). In case of legacy IEEE $802.11 \mathrm{a} / \mathrm{g}$ it is well known that there exists an optimal PHY mode [34], depending on the packet size and average SNR. Unfortunately, the transmitter requires the current average SNR in order to choose this optimal PHY mode. In case of comparison scheme 2, this knowledge can be assumed to be present at the station (due to the RTS/CTS handshake). In contrast, for comparison scheme 1 the transmitter does not know the current channel SNR and has to guess the optimal PHY mode. Alternatively, the transmitter could try to adapt the PHY mode to some average SNR experienced during previous transmissions to the same receiver. Nevertheless, in this study it is assumed that the transmitter can adapt the PHY mode optimally, as described qualitatively in [34]. Recall that this is a strong assumption in favor of the legacy mode, at least regarding comparison case 1 .

As we are primarily interested in the goodput data rate at the receiver, we require a model for the packet error probability. A prerequisite of the error model is that it must be applicable to the link adaptation case (i.e., legacy IEEE $802.11 \mathrm{a} / \mathrm{g}$ ) as well as to the adaptive modulation case (802.11 DYN). In our simulations we rely on an upper bound for the packet error probability, which takes the average bit error probability (of the modulation types per sub-carrier) as input. Note that in case of the adaptive modulation the system can control the bit error probability by setting the respective switching levels when to go from one modulation type to another one.

In $[16,33]$ an upper bound of the bit error probability is derived for binary convolutional coded transmission with hard-decision Viterbi decoding and independent bit 
errors. The resulting bit error probability is given by:

$$
P_{b} \leq 1 / k \sum_{d=d_{\text {free }}}^{\infty} c_{d} \cdot P_{d} .
$$

In this equation, $k$ is the number of input bits to the register of the convolutional encoder, $d_{\text {free }}$ is the free distance of the convolutional code, $P_{d}$ is the probability that an incorrect path of distance $d$ is chosen and $c_{d}$ is the number of bits in error in that case. The values for $c_{d}$ can be obtained by derivations; we have used the values from [23] for the rate $1 / 2$ coder with generator $(133,171)$. For the punctured rates with $3 / 4$ and $2 / 3$ we have used the corresponding values given in [27]. $P_{d}$ can be upper bounded as

$$
P_{d} \leq(2 \cdot \sqrt{\beta \cdot(1-\beta)})^{d} .
$$

In Equation 2, $\beta$ is the uncoded bit error probability of the OFDM physical layer. Given a certain modulation choice and a certain SNR per sub-carrier (either for link adaptation or for adaptive modulation), we calculate the uncoded bit error rate per sub-carrier and average over all $N$ bit error rate values (in case of 802.11 DYN the bit error probability of each sub-carrier has to be weighted by the modulation type in order to obtain the average). This average uncoded bit error rate is then applied as $\beta$ to Equation 2. The uncoded bit error rates are assumed to stay constant during the transmission of a packet. In order to obtain the bit error probability per subcarrier (given a certain SNR), we apply the formulas of [20] for coherent BPSK, QPSK, 16-QAM and 64-QAM under additive white Gaussian noise.

Given the bound on the resulting bit error probability $P_{b}$, we can obtain the packet error probability for a packet of size $\varsigma$ bits by:

$$
P_{p} \leq 1-\left(1-P_{b}\right)^{\varsigma}
$$

Notice that for high uncoded bit error probabilities (about 0.1 and larger), the bound of Equation 1 overestimates the resulting coded bit error probability [16] and hence a too high packet error probability is obtained. We correct this by introducing a scaling factor to the coded bit error probability, which is obtained by Lagrange interpolation (correcting the factor between simulated values [16] - which serve as reference - and the ones obtained from our analytical approach). Finally, we obtain a precise packet error probability model which allows to evaluate different packet sizes, different coding schemes and the two different physical layer approaches (link adaptation and adaptive modulation). We use this model for generating the packet error rates of any ongoing transmission-legacy IEEE 802.11a/g as well as 802.11 DYN.

All results are generated with OPNETmodeler Version 12.0.A-PL-5. Modifications of standard models required to support dynamic OFDM are with regard to the OPNET model library as of September 2006 [11]. For the simulation of the IEEE 802.11 system, we generally follow the standard as close as possible. In particu- 


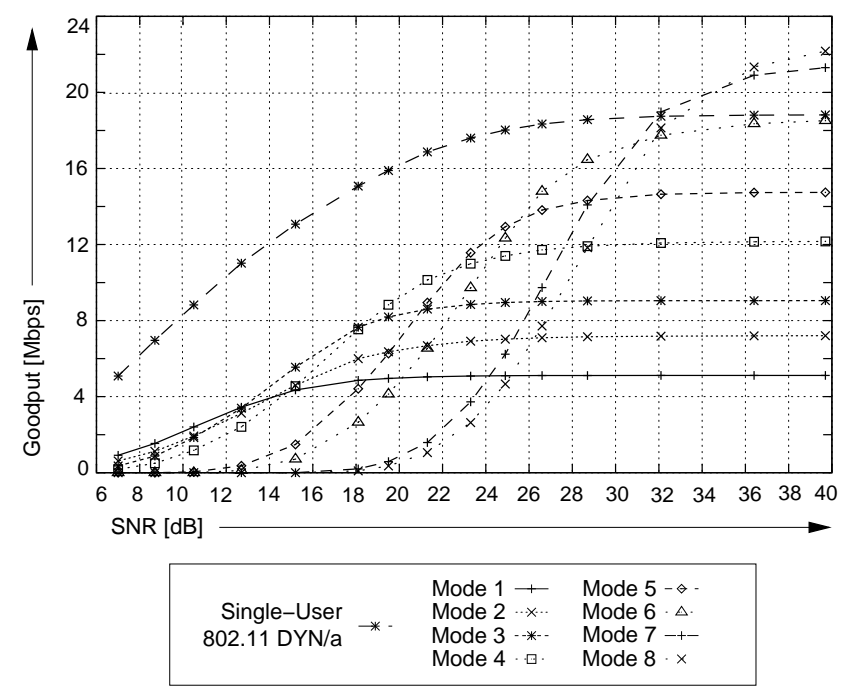

Fig. 5. Goodput comparison of 802.11 DYN/a and the eight legacy IEEE 802.11a PHY modes with RTS/CTS handshake for various different SNR levels and a MAC PDU size of 1564 Byte.

lar, we take the exponential backoff into consideration which the transmitter has to perform every time after transmitting a packet (if a station wants to re-access the WM immediately after finishing a packet transmission, it has to go into the exponential back-off according to the standard). Furthermore, we only consider long preambles. All non-payload frames (either for IEEE 802.11a or 802.11 DYN) are transmitted in base mode (BPSK with rate $1 / 2$ encoder). We only consider packet errors to occur in data frames. Hence, a retransmission is always due to an incorrect payload of the data frame. As stated above, we only consider a single transmitter and receiver (i.e., no collisions occur). For our investigations we vary the distance between transmitter and receiver (therefore we vary the average SNR) as well as the packet size. For a single simulation run we do not consider mobility.

\subsubsection{Results}

In Figure 5 we show the average goodput of 802.11 DYN/a versus the eight different legacy IEEE 802.11a PHY modes with RTS/CTS handshake. The shown results belong to a relatively large MAC SDU size of 1536 Byte plus the 28 Bytes for the IEEE 802.11 MAC overhead. Notice that at these large packet sizes an RTS/CTS frame exchange is normally performed in todays network cards of IEEE $802.11 \mathrm{a} / \mathrm{g}$. In case of the large packets, 802.11 DYN outperforms any legacy IEEE 802.11a PHY mode for any SNR point below $32 \mathrm{~dB}$. The performance difference is larger than $50 \%$ for many considered SNR points (we omit showing the confidence intervals as they are below one percent of deviation from the shown average values).

Where does this significant performance gain come from? Figure 6 and 7 present the average packet error rate and physical layer efficiency (payload bits-i.e., with- 


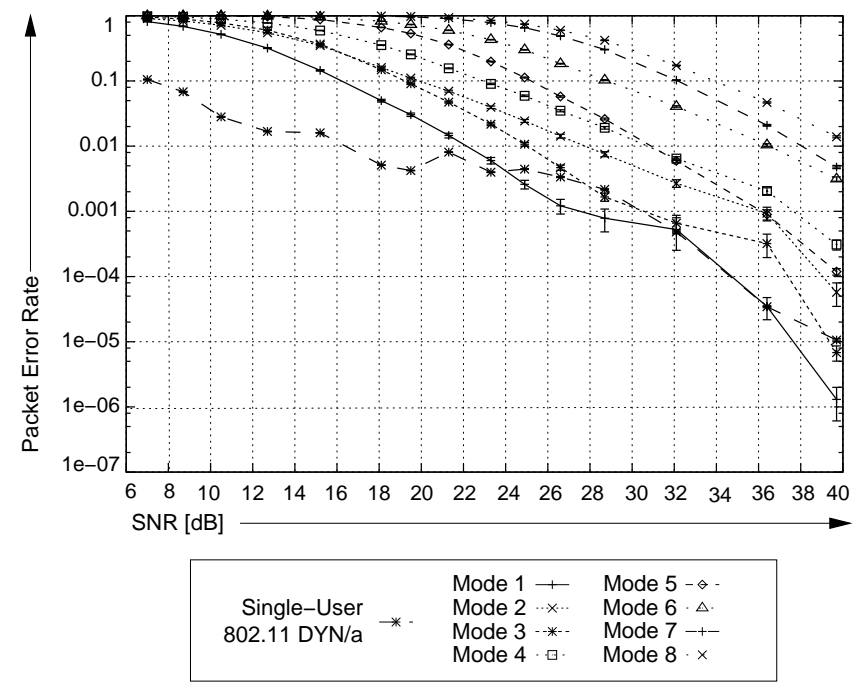

Fig. 6. Comparison of the packet error rate for all legacy IEEE 802.11a modes and 802.11 DYN/a (regarding a MAC PDU size of 1564 Byte and a varying SNR). The figure shows the packet error rates in logarithmic scaling.

out error correction bits-transmitted per sub-carrier per symbol) for 802.11 DYN/a and for all legacy IEEE 802.11a modes. The key "problem" of legacy OFDMbased IEEE 802.11 systems is the packet error rate of the link adaptation scheme. Employing the same modulation type on all sub-carriers creates a much higher bit error rate, as the fading always degrades the performance of a few sub-carriers severely. In contrast, these few badly fading sub-carriers can be simply "switched off" by adaptive modulation. This effect of switching them off leads even at a very high SNR to a PHY efficiency below 4.5 (meaning that even at high SNR not all sub-carriers are employed with 64-QAM and a convolutional coding rate of 3/4). In addition to the problem of the error rates, the PHY efficiency is also greater for 802.11 DYN/a up to $34 \mathrm{~dB}$ (as shown in Figure 7). A further difference between adaptive modulation and link adaptation is that the PHY efficiency increases steadily for adaptive modulation (in contrast to link adaptation).

In Figure 8 we show the average goodput results for smaller MAC PDU size of 228 Byte (including the 28 bytes added by the IEEE 802.11 MAC layer). Such packets occur for example in VoIP streams encoded according to G.711 with a bit rate of $64 \mathrm{kbps}$. Clearly, 802.11 DYN/a outperforms the legacy scheme significantly for an SNR range up to $20 \mathrm{~dB}$. However, the performance difference is much smaller than for large packets as the relative overhead in comparison to the payload length per packet transmission is now much higher.

In Figure 9 we show the corresponding results for the single-user 802.11 DYN/a mode versus legacy IEEE 802.11a without RTS/CTS. For small packets, the usage of the RTS/CTS handshake has a considerable impact on the performance. In this case the goodput difference is smaller but still significant for an SNR range up to 14 $\mathrm{dB}$. At an SNR of $16 \mathrm{~dB}$, mode 3 of legacy IEEE 802.11a achieves a better goodput 


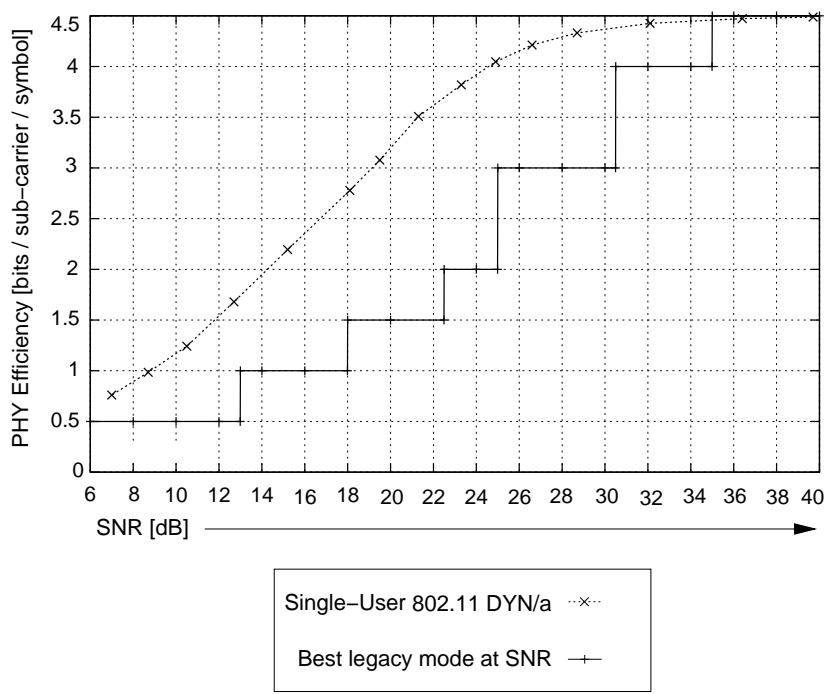

Fig. 7. PHY efficiency (in terms of bit per sub-carrier per symbol) for $802.11 \mathrm{DYN} / \mathrm{a}$ and the best performing legacy IEEE 802.11a mode at each SNR point respectively (i.e. the PHY mode which provides the highest goodput in Figure 5).

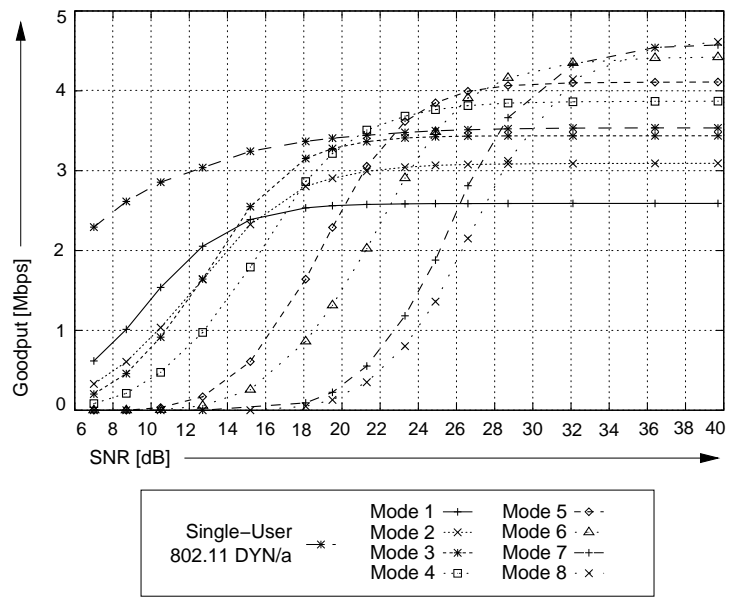

Fig. 8. Goodput comparison of 802.11 DYN/a and the eight legacy IEEE 802.11a PHY modes with RTS/CTS handshake for various different SNR levels and a MAC PDU size of 228 Byte.

and thereafter the legacy modes perform better. This is clearly due to the direct transmission of a packet without the RTS/CTS exchange. However, in such a case it is possible that the transmitter misses the correct mode to be used as the channel state is not known by the transmitter. Hence, in reality, we expect the goodput results to be lower for the legacy mode without RTS/CTS.

In both packet scenarios we observe that for low and medium SNR the dynamic OFDM approach performs better while for a medium to high SNR the legacy approach performs better. In Figure 10 we have plotted the SNR break even point for various packet sizes for the case with and without an RTS/CTS frame exchange. We observe that even for very small packet sizes, there still exists an SNR range for 


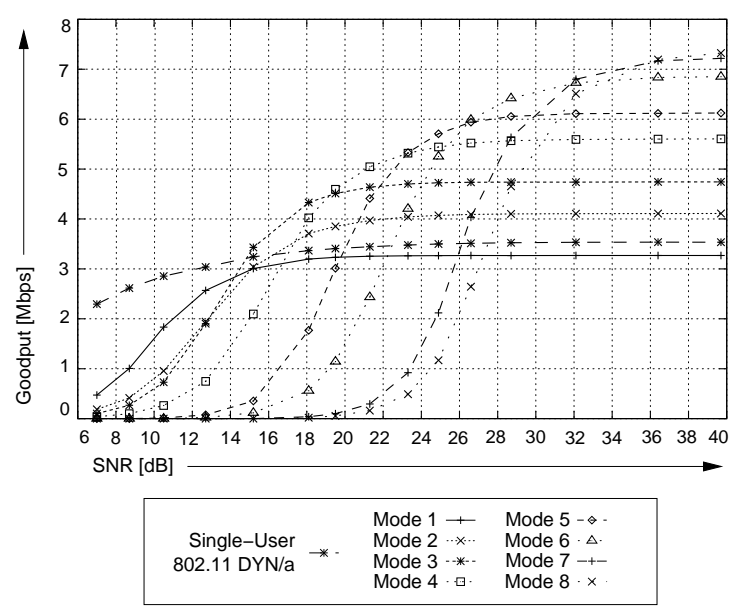

Fig. 9. Goodput comparison of 802.11 DYN/a and the eight legacy IEEE 802.11a PHY modes without RTS/CTS handshake for various different SNR levels and a MAC PDU size of 228 Byte.

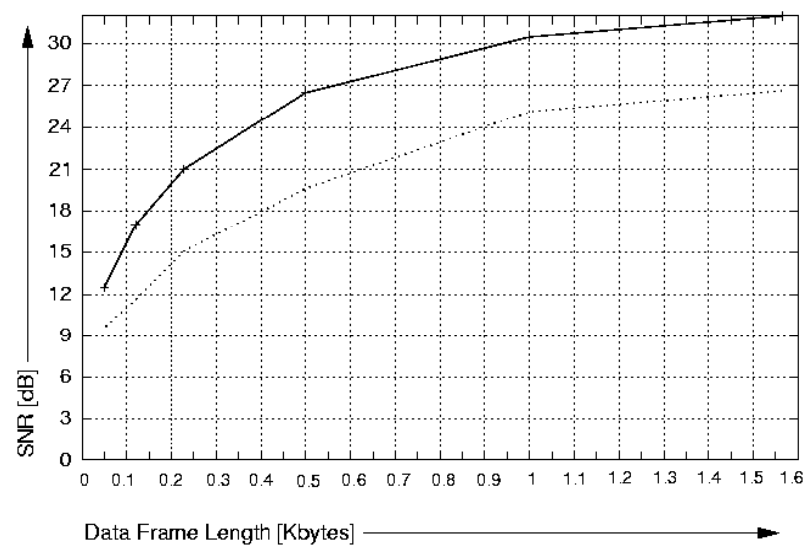

Fig. 10. SNR thresholds from which on legacy 802.11a outperforms 802.11 DYN/a. The solid line refers to 802.11a with RTS/CTS handshake, the dashed line corresponds to 802.11a without RTS/CTS handshake.

which 802.11 DYN/a outperforms legacy $802.11 \mathrm{a} / \mathrm{g}$. However, even for very large packet sizes 802.11 DYN/a is outperformed by 802.11a/g for an SNR larger than $30 \mathrm{~dB}$. In general, the impact of the RTS/CTS usage in case of $802.11 \mathrm{a}$ is about 3 to $5 \mathrm{~dB}$.

\subsection{Comparison of $802.11 \mathrm{DYN} / \mathrm{n}$ and $802.11 \mathrm{n}$}

Clearly, 802.11a/g is not the state-of-the-art in wireless LAN technology. This motivates us to also investigate the performance of dynamic OFDM schemes in 802.11n, the most recent (yet not officially ratified) amendment to the IEEE 802.11 standard. As briefly described in Section 2.3, 802.11n features a lot of improvements which cannot be covered all by this investigation. The most promising techniques, though, are frame aggregation at the link layer and MIMO transmission techniques at the 
physical layer. We select these two to be further investigated by us as described below.

\subsubsection{Simulation Model and Methodology}

Again we pick the average goodput as main metric for our investigation. The simulation setting is similar to the one used for the comparison in case of $802.11 \mathrm{a}$ (saturation mode, one transmitter, one receiver). The system parameters (like power, sub-carrier number and guard interval) are set according to 802.11n. Again we consider two packet sizes: large ones with a size of 1570 byte ( 6 more bytes - as the 802.11n MAC header is slightly larger) as well as small ones of size 234 byte. Regarding the frame aggregation, we pick the A-MPDU method as it allows for the correction of single packets without retransmitting the whole aggregated frame (thus the simulation contains the block acknowledgment feature of 802.11n). Note that although we consider frame aggregation, we restrict it for the specific packet sizes to certain values. In case of large packets (of size 1570 byte) we set the frame aggregation to a maximum of 4 PDUs, as the wireless channel cannot be assumed to be stable in case that more (large) packets are aggregated. For small packets the limiting factor is the delay: we anticipate VoIP traffic in case of a packet size of 234 byte. Thus, sequential packets of a certain stream have approximately an interarrival time of $20 \mathrm{~ms}$, such that an aggregation of 4 packets leads to an additional delay of $80 \mathrm{~ms}$ for the packet that arrived first to the access point. We consider this as upper limit. Finally, for the MIMO transmission system we only consider the case of spatial multiplexing with a 2 by 2 antenna setting.

Again, we employ OPNETmodeler Version 12.0.A-PL-5 for our investigations with an appropriately modified version of the model library as of September 2006 [11]. Regarding the packet error generation, we use the same model as in case of our 802.11a performance evaluation, but of course applied now to a much larger number of sub-carriers. In order to generate the channel matrix (as a 2 by 2 MIMO system is considered, the channel is no longer a single attenuation value but a matrix), the 802.11n task group published a MATLAB module to generate traces of MIMO channel states [35,22]. We use this tool to generate the channel matrix and consider on top of this an MMSE receiver for decoupling the spatial streams. Regarding the channel matrix generation, we consider channel type 'E' representing a large office environment with a delay spread of 100ns [22].

The performance evaluation starts for large packets (Section 4.2.2) - hence having RTS/CTS handshake enabled for 802.11n. First we evaluate "plain 802.11n", i.e., disabling frame aggregation and spatial multiplexing. Afterwards, we consecutively add spatial multiplexing, frame aggregation, and the combination of the two. We abstain from plotting 95\% confidence intervals for illustrative purposes as they are below one percent of deviation from the plotted average. Results for small packets are presented afterwards in the same order (Section 4.2.3. In this case RTS/CTS 


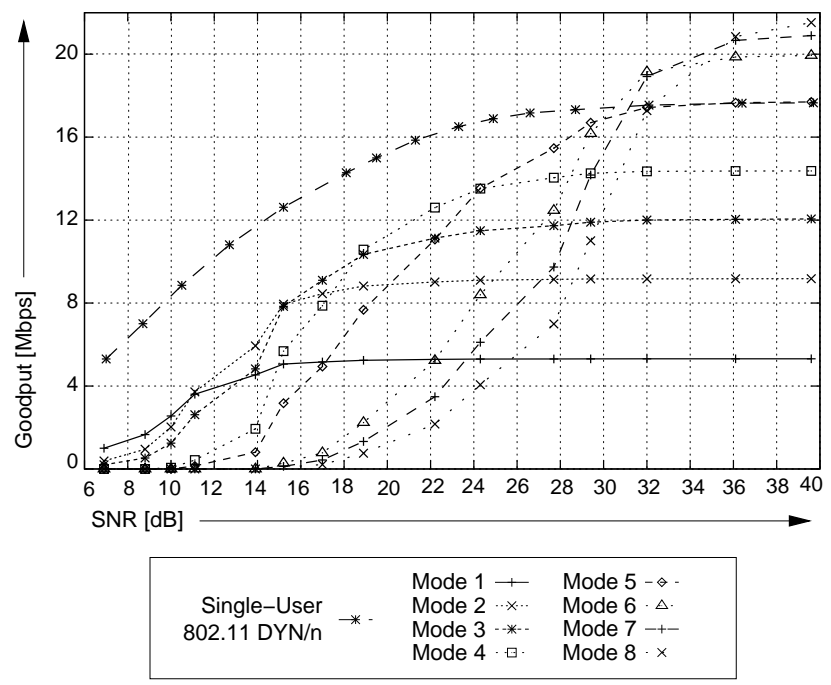

Fig. 11. Goodput comparison of 802.11n and 802.11 DYN/n - Scenario: Large packets, RTS/CTS active, no frame aggregation, no spatial multiplexing.

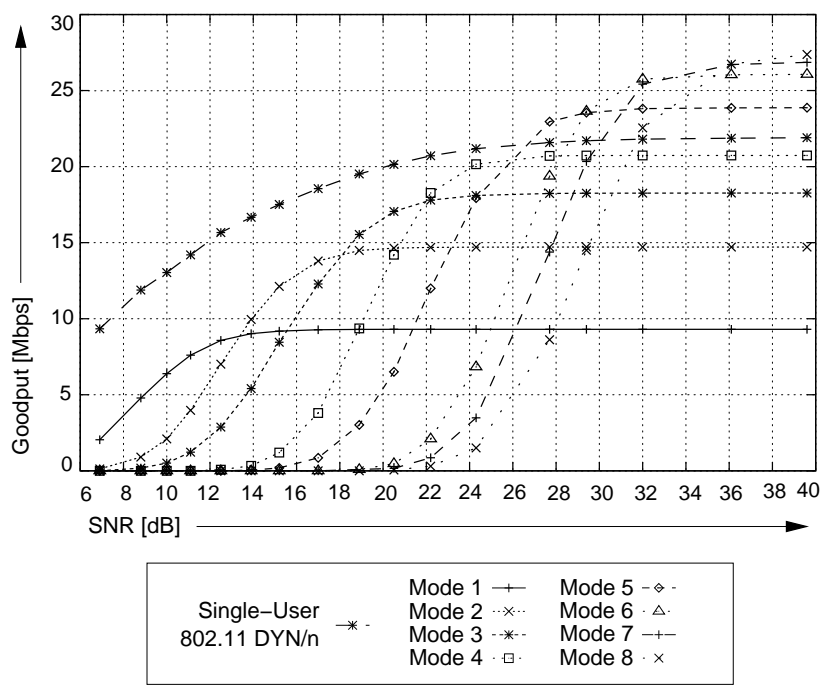

Fig. 12. Goodput comparison of 802.11n and $802.11 \mathrm{DYN} / \mathrm{n}$ - Scenario: Large packets, RTS/CTS active, no frame aggregation, 2 by 2 antenna spatial multiplexing.

handshake is only activated if frame aggregation is also active.

\subsubsection{Results - Large Packets}

Figure 11 shows that $802.11 \mathrm{DYN} / \mathrm{n}$ provides a significant (about 50\%) performance gain for all SNRs below $30 \mathrm{~dB}$ as compared to $802.11 \mathrm{n}$. This performance gain decreases slightly if only spatial multiplexing is added, as shown in Figure 12 for a 2x2 MIMO system. Notably, 802.11 DYN/n now outperforms 802.11 n only up to an SNR of about $26 \mathrm{~dB}$, thereafter $802.11 \mathrm{n}$ provides a higher throughput.

Next we consider adding only frame aggregation, thus deactivating spatial multi- 


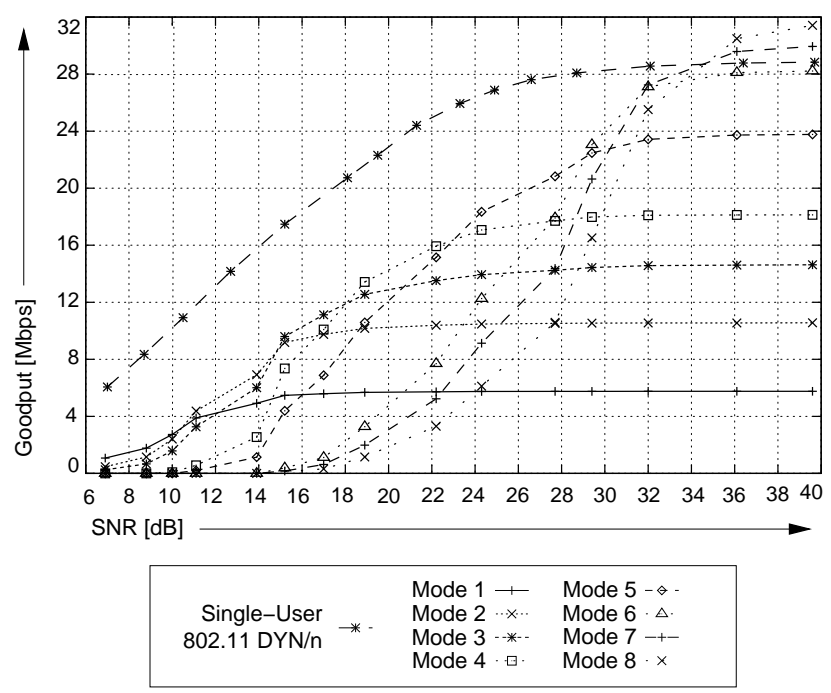

Fig. 13. Goodput comparison of $802.11 \mathrm{n}$ and $802.11 \mathrm{DYN} / \mathrm{n}$ - Scenario: Large packets, RTS/CTS active, frame aggregation of 2 packets, no spatial multiplexing.

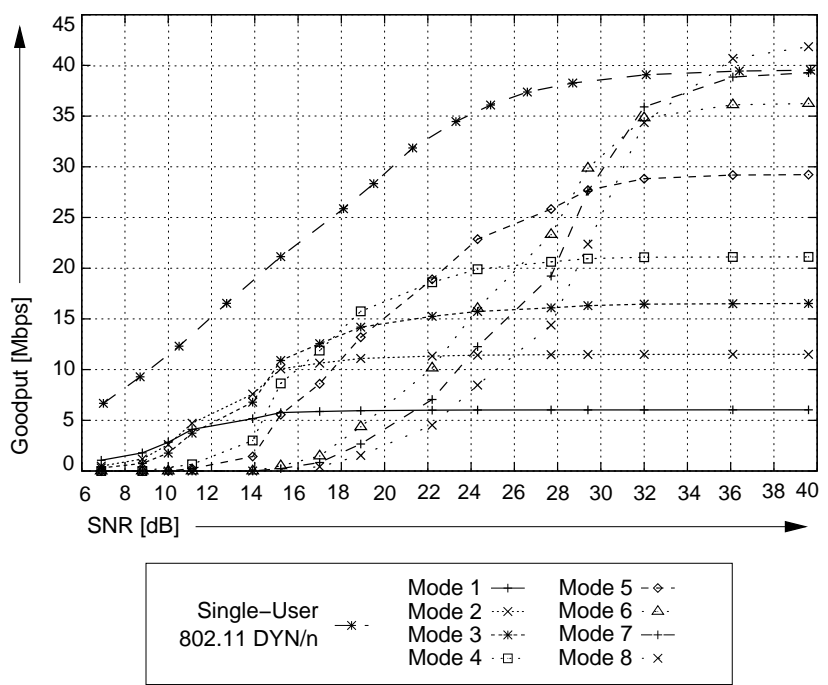

Fig. 14. Goodput comparison of $802.11 \mathrm{n}$ and $802.11 \mathrm{DYN} / \mathrm{n}^{\prime}-$ Scenario: Large packets, RTS/CTS active, frame aggregation of 4 packets, no spatial multiplexing.

plexing. The corresponding results are shown in Figure 13 for an aggregation of 2 packets and in Figure 14 for an aggregation of 4 packets. Clearly, 802.11 DYN/n benefits stronger from frame aggregation as $802.11 \mathrm{n}$ does, since the payload transmission phase (during which DYN achieves a higher spectral efficiency and a better packet error rate) is now much longer. Hence, the increase of overhead becomes less an issue. 802.11 DYN/n provides even at high SNR a comparable performance to 802.11n (while outperforming by $100 \%$ and more for smaller SNRs). Figures 15 and 16 illustrate the effect of combining both, spatial multiplexing and frame aggregation. Whereas with frame aggregation, $802.11 \mathrm{n}$ was almost always outperformed by $802.11 \mathrm{DYN} / \mathrm{n}$ (c.f. Fig. 12), the activation of spatial multiplexing decreases this performance gap slightly (as is also the case if no frame aggregation is considered 


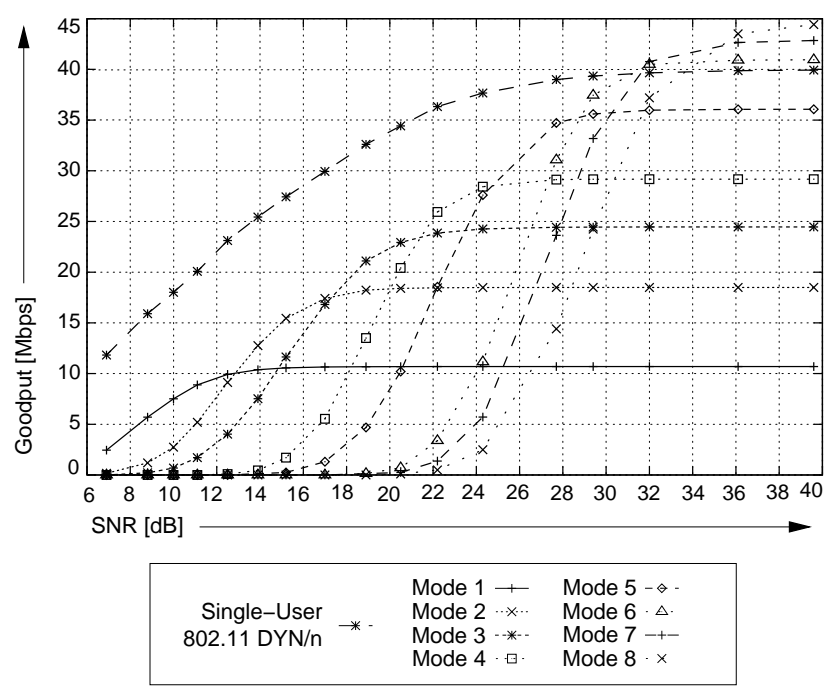

Fig. 15. Goodput comparison of 802.11n and 802.11 DYN/n - Scenario: Large packets, RTS/CTS active, frame aggregation of 2 packets, 2 by 2 antenna spatial multiplexing.

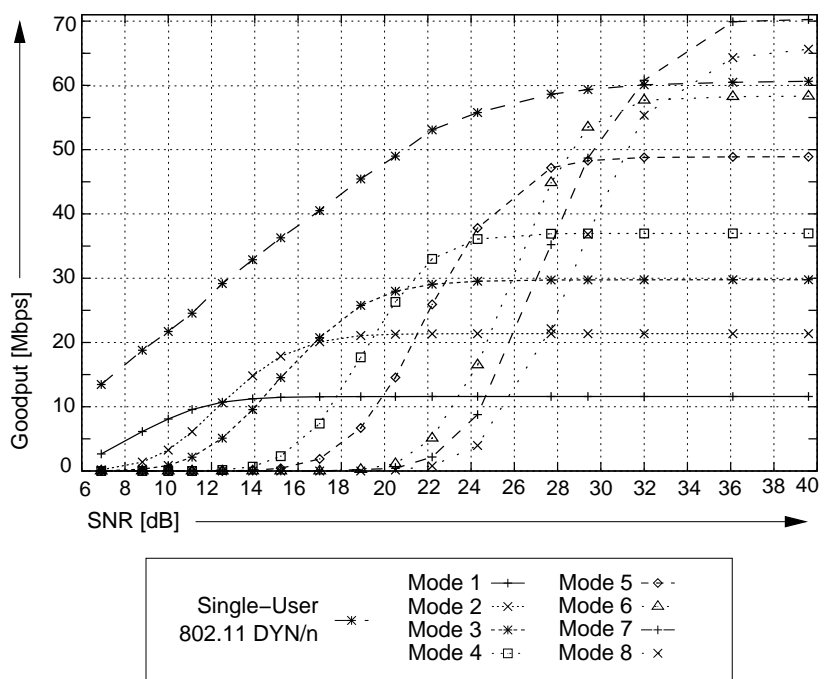

Fig. 16. Goodput comparison of 802.11n and 802.11 DYN/n - Scenario: Large packets, RTS/CTS active, frame aggregation of 4 packets, 2 by 2 antenna spatial multiplexing.

in Figure 11 and 12), as spatial multiplexing leads to an increase of overhead (i.e. signaling) for $802.11 \mathrm{DYN} / \mathrm{n}$ while this is not the case for $802.11 \mathrm{n}$.

\subsubsection{Results - Small Packets}

For small packets, the performance gain of $802.11 \mathrm{DYN} / \mathrm{n}$ is not as impressive as for large packets. In fact, for a 'plain' 802.11n system (without frame aggregation and without spatial multiplexing), 802.11 DYN/n does not pay off at all for smaller packets in comparison to $802.11 \mathrm{n}$ without RTS/CTS handshake. The reason for this is the additional overhead introduced for $802.11 \mathrm{n}$ in general, especially for the preamble. As 802.11 DYN/n is based on five frames (RTS, CTS, payload, 


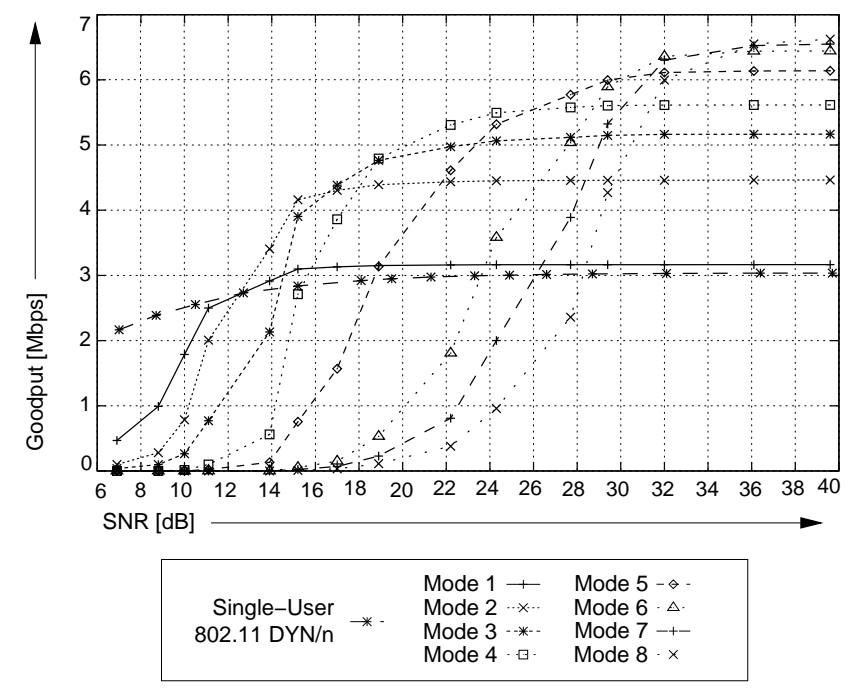

Fig. 17. Goodput comparison of 802.11n and 802.11 DYN/n - Scenario: Small packets, no RTS/CTS, no frame aggregation, no spatial multiplexing.

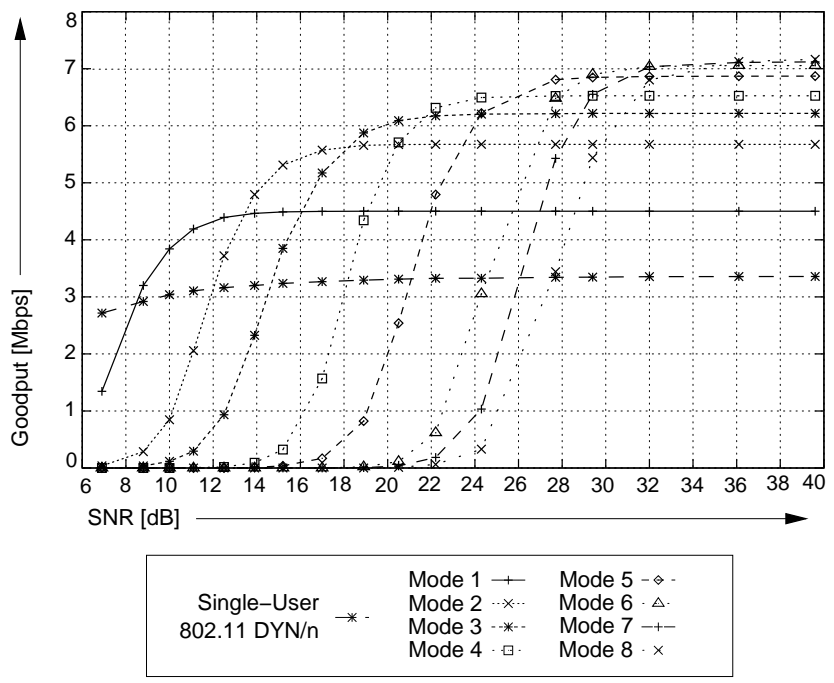

Fig. 18. Goodput comparison of 802.11n and 802.11 DYN/n - Scenario: Small packets, no RTS/CTS, no frame aggregation, 2 by 2 antenna spatial multiplexing.

ACK, and CTS-to-self), the new preamble overhead becomes the dominant factor in the performance (as shown in Figure 17 recall that the new, longer preamble has to be transmitted prior to each of the five frames in case of $802.11 \mathrm{DYN} / \mathrm{n}$ while we compare it here to $802.11 \mathrm{n}$ without RTS/CTS handshake which only requires two longer preamble). 802.11 DYN/n provides only for very small SNRs a performance improvement. Adding a second spatial stream makes this situation even worse, as it primarily adds overhead for signaling in case of $802.11 \mathrm{DYN} / \mathrm{n}$. Effectively, 802.11 DYN/n provides a lower performance for any SNR larger than $8 \mathrm{~dB}$ as shown in Figure 18. However, one should recall that we always assume an optimal rate selection in case of $802.11 \mathrm{n}$ even if no RTS/CTS handshake is performed. Hence, the application of realistic rate selection algorithms certainly decreases the performance of $802.11 \mathrm{n}$. 


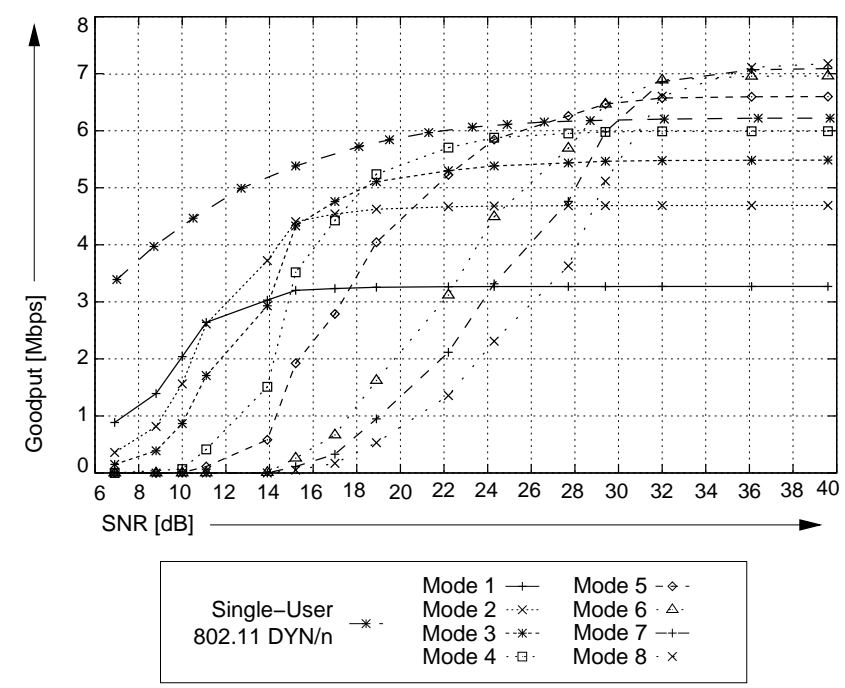

Fig. 19. Goodput comparison of 802.11n and 802.11 DYN/n - Scenario: Small packets, RTS/CTS active, frame aggregation of 2 packets, no spatial multiplexing.

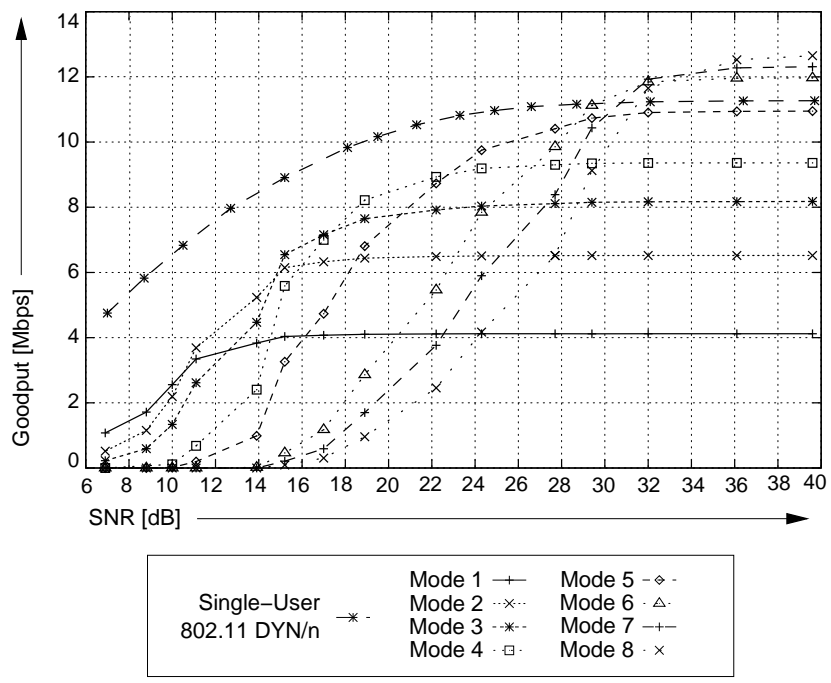

Fig. 20. Goodput comparison of $802.11 \mathrm{n}$ and $802.11 \mathrm{DYN} / \mathrm{n}$ - Scenario: Small packets, RTS/CTS active, frame aggregation of 4 packets, no spatial multiplexing.

Next, we consider the activation of frame aggregation without spatial multiplexing for two aggregated MAC PDUs (Figure 19) and for four MAC PDUs (Figure 20). Note that due to the increased payload size we consider now the usage of the RTS/CTS handshake in case of 802.11n. Consequently, as the performance of 802.11 DYN/n is stronger improved by frame aggregation, the performance difference changes in this situation. 802.11 DYN/n outperforms now $802.11 \mathrm{n}$ for low and medium SNRs up to 26 or even $30 \mathrm{~dB}$. Note that the performance gain is about $50 \%$ or larger. The reason for this different situation is the same as in the case of large packets: the larger the payload in comparison to the overhead, the better performs 802.11 DYN/n. Finally, in Figures 21 and 22 we consider the activation of frame aggregation and spatial multiplexing for the two different numbers of aggregated MAC PDUs. In general, adding spatial multiplexing to frame aggregation leads to a 


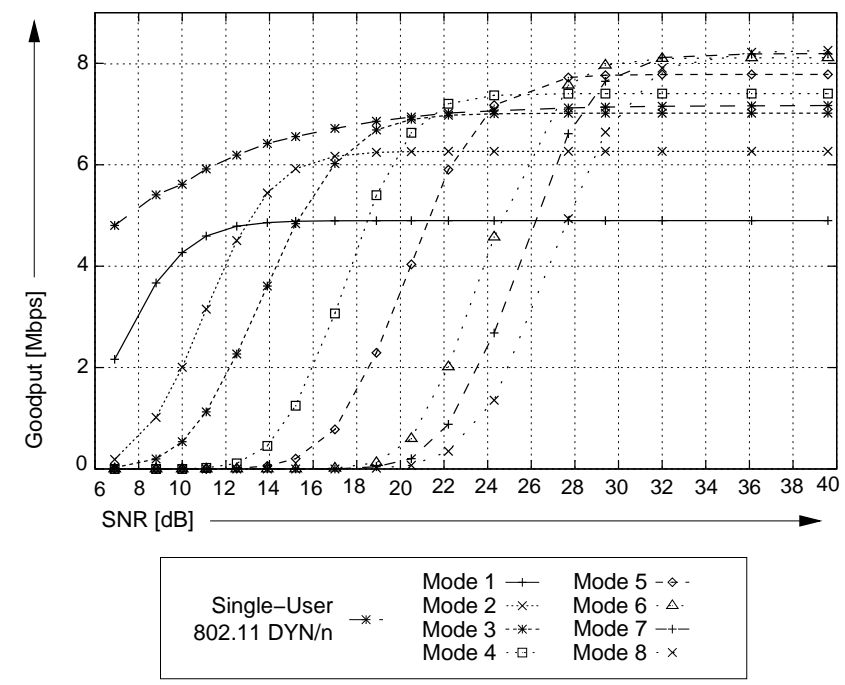

Fig. 21. Goodput comparison of 802.11n and 802.11 DYN/n - Scenario: Small packets, RTS/CTS active, frame aggregation of 2 packets, 2 by 2 antenna spatial multiplexing.

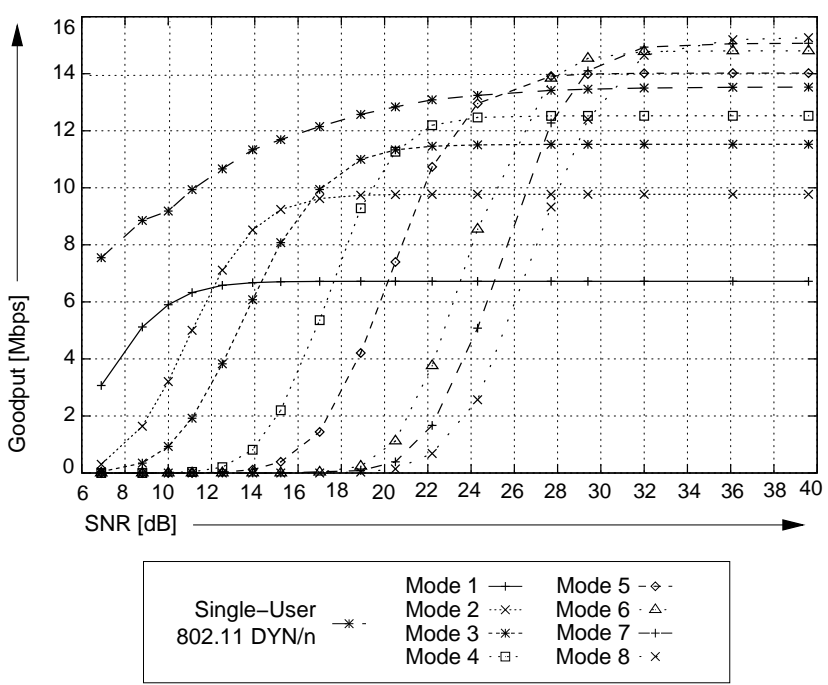

Fig. 22. Goodput comparison of 802.11n and 802.11 DYN/n - Scenario: Small packets, RTS/CTS active, frame aggregation of 4 packets, 2 by 2 antenna spatial multiplexing.

higher overhead for 802.11 DYN/n such that the performance gain between 802.11 DYN/n and 802.11n decreases. However, 802.11 DYN/n still provides a significant performance gain for low to medium SNRs.

\section{Conclusions and Future Work}

In this paper we have presented a protocol extension to legacy IEEE 802.11a/g and its emerging amendment $802.11 \mathrm{n}$ enabling the dynamic adaptation of the modulation type per sub-carrier to the current channel gain. This requires the transmitter to 
acquire channel state information while the receiver has to be informed of the used modulation type per sub-carrier. We suggest to start each such transmission with an RTS/CTS handshake (used to estimate the sub-carrier gains) while extending the PLCP frame for the payload data transmission to carry signaling information as well. Evaluating this scheme by simulations, we show that the new approach significantly outperforms the legacy IEEE 802.11a as well as IEEE 802.11n, even if the legacy mode is not using the RTS/CTS handshake. Especially for large packet sizes the performance difference is quite large. We argue that this is due to a much better control of the frequency selective channel, leading to a (slightly) physical layer efficiency (in terms of average payload bits transmitted per sub-carrier per modulation symbol) and a (significantly) lower packet error rate. The results show that even though emerging WLAN systems, i.e., IEEE 802.11n, employ advanced MIMO technology and hence enable several spatial streams, dynamic adaptation of the modulation type per sub-carrier can still increase the system performance.

As future work we consider the application of dynamic OFDM multi-user schemes in 802.11 DYN such that several stations are served simultaneously by the access point. While benefiting from the better control of the channel and an even higher throughput (due to exploiting the multi-user diversity) such an approach has a lot of potential from the link layer perspective as well, as only one channel access has to be performed for the transmission of several packets (hence, the link layer efficiency will increase, too).

\section{Acknowledgments}

The authors would like to thank OPNET Technologies, Inc., for providing the simulation environment under the OPNET University Program.

\section{References}

[1] Ieee 802.11 - wireless lan medium access control (mac) and physical layer (phy) specifications (1999).

[2] Ieee $802.11 \mathrm{~b}$ - high speed physical layer extension in the $2.4 \mathrm{ghz}$ band, amandment 2 to 802.11 (Sep. 16 1999).

URL http://standards.ieee.org/getieee802/download/802. $11 b-1999 \cdot \mathrm{pdf}$

[3] Ieee $802.11 \mathrm{a}$ - high-speed physical layer in the $5 \mathrm{ghz}$ band, amandment 1 to 802.11 (2000).

URL http://standards.ieee.org/getieee802/download/802. $11 a-1999 \cdot \mathrm{pdf}$ 
[4] Ieee 802.11f - trial-use recommended practice for multi-vendor access point interoperability via an inter-access point protocol across distribution systems supporting ieee 802.11 (June 12 2003).

URL http://standards.ieee.org/getieee802/download/802. $11 \mathrm{~F}-2003 \cdot \mathrm{pdf}$

[5] Ieee $802.11 \mathrm{~g}$ - further higher data rate extension in the $2.4 \mathrm{ghz}$ band, amandment 4 to 802.11 (June 27 2003).

URL http://standards.ieee.org/getieee802/download/802. $11 \mathrm{~g}-2003 \cdot \mathrm{pdf}$

[6] Ieee 802.11i - a medium access control (mac) security enhancements (June 24 2004).

[7] Ieee 802.11e - medium access control (mac) quality of service (qos) enhancements (January 2005).

[8] Ieee 802.11revma d5.1 - wireless lan medium access control (mac) and physical layer (phy) specifications (2006).

[9] Ieee 802.11 - wireless lan medium access control (mac) and physical layer (phy) specifications (June 12 2007).

[10] Ieee $802.11 \mathrm{n}$ - enhancements for higher throughput, amendment 4 to ieee 802.11 part 11: Wireless lan medium access control (mac) and physical layer (phy) specifications (December 1 2007).

[11] Opnet modeler wireless (2007).

URL www. opnet.com/products/modeler

[12] E. E. . 744, Digital Video Broadcasting (DVB); Framing Structure, Channel Coding, and Modulation for Digital Terrestial Television (August 1997).

[13] A. Aguiar, J. Klaue, Bi-directional WLAN Channel Measurements in Different Mobility Scenarios, in: Proc. of Vehicular Technology Conference (VTC Spring), Milan, Italy, 2004.

[14] J. Bingham, Multicarrier Modulation for Data Transmission: An Idea Whose Time Has Come, IEEE Commun. Mag. 28 (1990) 5-14.

[15] M. Bohge, J. Gross, M. Meyer, A. Wolisz, Dynamic Resource Allocation in OFDM Systems: An Overview of Cross-Layer Optimization Principles and Techniques, IEEE Network 21 (1) (2007) 53-59.

[16] M. Bossert, Channel Coding for Telecommunications, John Wiley \& Sons, Inc., 2000.

[17] J. Campello, Practical bit loading for DMT, in: Proc. IEEE Int. Conference on Communications (ICC), 1999.

[18] J. Cavers, Mobile Channel Characteristics, chap. 1.3, Kluwer Academic, 2000.

[19] R. Chang, R. Gibby, A Theoretical Study of Performance of an Orthogonal Multiplexing Data Transmission Scheme, IEEE Trans. Commun. Technol. COM-16 (1968) 529-540. 
[20] K. Cho, D. Yoon, On the General BER Expressions of One- and Two-Dimensional Amplitude Modulations, IEEE Trans. Commun. 50 (7) (2002) 1074-1080.

[21] A. Czylwik, Adaptive OFDM for Wideband Radio Channels, in: Proc. of the Global Telecommunications Conference, 1996.

[22] V. Erceg, L. Schumacher, P. Kyritsi, A. Molisch, D. S. Baum, A. Y. Gorokhov, C. Oestges, Q. Li, K. Yu, N. Tal, N. Bas Dijkstra, A. Jagannatham, C. Lanzl, V. J. Rhodes, J. Medbo, D. Michelson, M. Webster, Tgn channel models (May 2004).

[23] P. Frenger, P. Orten, T. Ottoson, Convolutional Codes with Optimum Distance Spectrum, IEEE Trans. Commun. 3 (11) (1999) 317-319.

[24] J. Gross, M. Emmelmann, O. Puñal, 802.11 DYN: Protocol Extension for the Application of Dynamic OFDM(A) Schemes in 802.11a/g Systems, Tech. Rep. TKN07-002, Telecommunication Networks Group, Technische Universität Berlin (May 2007).

[25] J. Gross, M. Emmelmann, O. Puñal, A. Wolisz, Dynamic Point-to-Point OFDMA Adaptation for IEEE 802.11a/g Systems, doc. 11-07/720, IEEE 802.11 WNG SC Wireless Next Generation Standing Committee, Montreal, Canada (May 14 - 18 2007).

[26] J. Gross, M. Emmelmann, O. Punal, A. Wolisz, Dynamic single-user ofdm adaptation for ieee 802.11 systems, in: Proc. ACM/IEEE International Symposium on Modeling, Analysis and Simulation of Wireless and Mobile Systems (MSWIM 2007), Chania, Crete Island, 2007.

[27] D. Haccoun, G. Begin, High-Rate Punctured Convolutional Codes fro Viterbi and Sequential Decoding, IEEE Trans. Commun. 37 (11) (1989) 1113-1125.

[28] D. Hughes-Hartogs, Ensemble Modem Structure for Imperfect Transmission Media, u.S. Patents 4,679,227 (July 1987); 4,731,816 (March 1988); 4,833,706 (May 1989).

[29] H. Liu, G. Li, OFDM-Based Broadband Wireless Networks, John Wiley \& Sons, Inc., 2005.

[30] H. Nguyen, J. Brouet, V. Kumar, T. Lestable, Compression of Associated Signaling for Adaptive Multi-Carrier Systems, in: Proc. Vehicular Technology Conference (VTC Spring), 2004.

[31] B. O’Hara, A. Petrick, IEEE 802.11 Handbook, 2nd ed., IEEE Press, 2005.

[32] L. Piazzo, Fast Optimal Bit-Loading Algorithm for Adaptive OFDM Systems, Tech. Rep. 002-04-03, University of Rome (2003).

[33] J. Proakis, Digital Communications, McGraw-Hill, 1995.

[34] D. Qiao, S. Choi, Goodput Enhancement of IEEE 802.11a Wireless LAN via Link Adaptation, in: Proc. IEEE Int. Conference on Communications (ICC), 2001.

[35] L. Schumacher, Wlan mimo channel matlab program, Tech. rep.

URL http://www.info.fundp.ac.be/ 1sc/Research/IEEE_80211_ HTSG_CMSC/distribution_terms.html 